\title{
PREDICTION OF THE CUTTING DEPTH OF ABRASIVE SUSPENSION JET USING A BP ARTIFICIAL NEURAL NETWORK
}

\author{
Xiaojian Liu ${ }^{1,2}$, Tao Yu', Wenbin Wang ${ }^{1}$ \\ ${ }^{1}$ CIMS \&Robot Center of Shanghai University, Shanghai 200072, China. ${ }^{2}$ Shandong Institute \\ of Light Industry Shandong 250100, China.Email: lxj@sdili.edu.cn.
}

\begin{abstract}
Abrasive suspension jet is a new embranchment of abrasive jet. In this paper, the abrasive suspension technology is first used in cutting process in domestic market. The abrasive grain concentration in suspension is constant, so the cutting quality is more stable. In this paper, a prediction model based on a back-propagation (BP) artificial neural network is presented for predicting the cutting depth generated by abrasive suspension jet. In the application of the BP neural network, the mean error of the output in the model training is 0.01 , the relatively discrepancy is below $8.70 \%$. The modeling method based on the BP neural network is much more convenient and exact compared with traditional methods, and can always achieve a much better prediction effect. It is verified with experiments to be reasonable and feasible, and it is the better foundation for the future study of abrasive suspension jet.
\end{abstract}

Key words: neural network; abrasive suspension jet; cutting depth

\section{INTRODUCTION}

Abrasive water jet (AWJ) machining has been accepted in the industry due to several factors, among which are its versatility for use on a wide range of materials and the high-quality surfaces produced. These surfaces are free from heat affected zones(HAZ)and mechanical distortions. however, AWJ machining has some limitations:

Please use the following format when citing this chapter:

Liu, Xiaojian, Yu, Tao, Wang, Wenbin, 2006, in International Federation for Information Processing (IFIP), Volume 207, Knowledge Enterprise: Intelligent Strategies In Product Design, Manufacturing, and Management, eds. K. Wang, Kovacs G., Wozny M., Fang M., (Boston: Springer), pp. 563-569. 
- Relatively slow cutting rates when compared to laser(when HAZ is not critical).

- Limited in cutting thin kerfs.

- When used with garnet abrasives, not efficient for cutting carbides, ceramics, and hard materials.

As the need for improved cutting and machining capabilities increases, more attention is being focused on improving the above limitations of the AWJ. Work is underway to introduce an alternative technique to complement the AWJ process capabilities without sacrificing any of its advantages, so abrasive suspension is proposed. It's an embranchment of abrasive jet in theory. In this paper, abrasive suspension jet (ASJ) is concerned. The advantages of this cutting technology is:

- The energy transfer to particles is more efficient

- Nozzles can be miniaturized to fit in tight spaces.

- The ASJ can machine thinner kerfs and smaller holes than AWJ.

The influence factors on cutting ability of low-pressure abrasive suspension jet are complex, they often have nonlinear relations with each other. It's difficult to propose a mathematics model of cutting depth exactly, and a model can be set based on neural network. Unlike the traditional method, the artificial neural network (ANN) does not require a mathematical model. ANN can perform not only a highly accurate non-linear, but also retains memory of data. Further, the ANN is powerful in handling dispersed data, and moreover it can also adjust the state of the network on the base of the original network in order to adapt to new data- sets through training with these new data- sets. In this paper, an ANN is used in predicting the cutting depth and the optimum structure of the ANN is also proposed.

\section{THE BASIC PRINCIPLE OF ARTIFICIAL NEURAL NETWORK}

An artificial neural network has multi-layers. There is an input layer where data are presented to the neural network, and an output layer that holds the response of the network to the input. It is the intermediate layers, also known as hidden layers, that enable these network to represent and compute complicated associations between patterns. Neuron is the basic element. Each neuron is connected to all the neurons in the next layer.

For a training pattern $\left(X_{k}, Y_{k}\right)(k=1,2, \ldots N)$ in which $X_{k}$ is the input pattern and can be expressed as an no-dimensional vector $\left(x_{k 1}, x_{k 2}, \ldots x_{k n}\right)^{T}$ and $Y_{k}$ is the corresponding expected solution and can be expressed as an nodimensional vector $\left(y_{k 1}, y_{k 2}, \ldots y_{k m}\right)^{T}$, the net input to a neuron is usually computed as the sum of all inputs to it: 
Artificial Neural Network

$$
\mathrm{I}_{k j}^{l}=\sum_{i} W_{j i}^{l} O_{k i}^{l-1}+\theta_{j}^{l}
$$

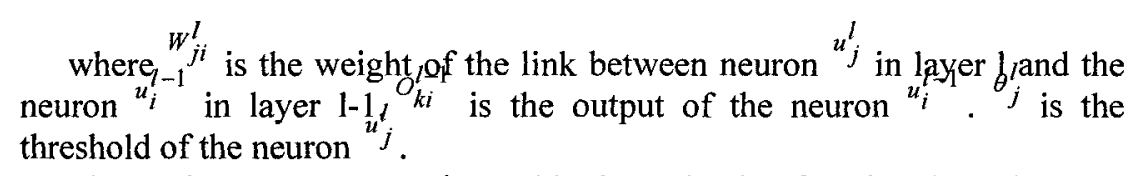

The net input to a neuron is used by its activation function, here the most commonly used sigmoid function, to produce an output

$$
o_{k j}^{l}=f\left(I_{k j}^{l}\right)=\left[1+\exp \left(-I_{k j}^{l}\right)\right]^{-1}
$$

where $f($.$) is the transfer function. The transfer function has several forms,$ such as the hyperbolic tangent, step, or sigmoid function. However , the sigmoid function is the most common in practical application and is used here.

The BP neural network is characterized by gaining possession of hidden layers. Generally, a BP network with one hidden layer is enough for most applications, and one more hidden layers tends to make the network too complicated, a potential that results in more minimums and causes lower convergence speeds and larger errors. Theoretically, BP with two hidden layers is enough to explain the complicated system with more general classic boundaries and has faster convergence speeds.

In the BP learning scheme, the calculated outputs in the output layer, ${ }^{\prime}{ }^{\prime} j$ are compared with the desired outputs ${ }^{y} k j$, to find the error, before the error signals are propagated backward through the network. The error function $\mathrm{E} \mathrm{k}$ can be defined as

$$
E_{k}=\frac{1}{2} \sum_{j=1}^{m}\left(y_{k j}-y_{k j}^{\prime}\right)^{2}
$$

where $m$ is the total number of the output neurons.

In the BP algorithm, each training pattern is presented once and the weight correction is calculated, but the weights are not actually adjusted; calculated weight corrections for each weight are added together for all the patterns and then weights are adjusted only once using the cumulative correction. The momentum strategy implements a variable learning rate coefficient implicitly by adding a fraction of movement in the weight change to the current direction of movement in the weight apace. The new equation for weight change is given by 


$$
W_{j i}^{l}[T+1]=W l_{j i}^{l}-\eta\left(\frac{\partial E}{\partial W_{j i}^{l}[T]}\right)+\alpha \times\left(W_{j i}^{l}[T]-W_{j i}^{l}[T-1]\right)
$$

where $\eta$ is the step size or the learning rate coefficient, the index $T$ labels the iteration number in the learning process, and $\alpha$ is a momentum coefficient that can have a value between 0 and 1 .

\section{THE PREDICTION OF CUTTING DEPTH BY NEURAL NETWORK}

\subsection{Experiments of cutting depth}

There are many factors that influence the cutting depth of abrasive suspension jet. The dominating factors are cutting pressure, feed rate, additive concentration and standoff distance. So in this paper, four factors are in considered. The experiment conditions are: grain mesh: 80,grain type :garnet, cutting material: A3 steel, cutting angle: $90^{\circ}$.

\subsection{Determination of the structure of the BP network}

In order to establish the process model, we first need to decide how many layers and how many neurons per layer of the network are utilized to describe the behavior of cutting. It is easy to determine the number of neurons in the input layer and the output layer during applications. There are four neurons in the input layer denoting the content of cutting pressure, feed rate, abrasive flow rate, additive concentration, respectively, and one neuron in the output layer representing the corresponding the cutting depth. However it is not so easy to choose the appropriate number of neurons in the hidden layer for there is currently no definite rule to determining it except through experimentation. Using too few neurons impairs the neural network and prevents the correct mapping of input to output. Using too many neurons impedes generalization and increases training time. In our study, ten hidden neurons are finally employed in the BP network and the structure of this neural network is shown in Figure 1. 


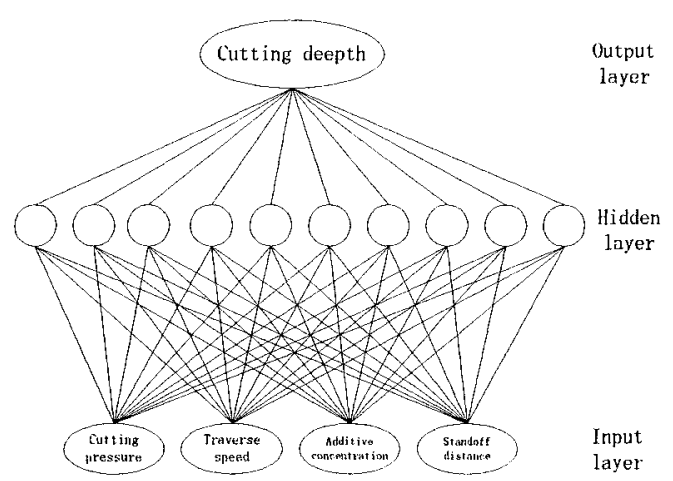

Figure 1. The depth prediction model of BP neural network

\subsection{Choice of training precision}

In the $\mathrm{BP}$ algorithm, training is carried out by repeatedly presenting the entire set of training patterns (with the weights updated at the end of each cycle) until the energy functions over all the training patterns are minimized and within the preset training precision. In principle, the BP network can always remember all the training patterns after enough large iterations. But what should the training precision be set to terminate the training course? in actual engineering systems the training data are usually erroneous, so the too high training precision will over fit the training patterns and then impede generalization of the BP network. The training precision of this paper is determined with 0.01 .

\subsection{Network training and verification}

Data of 20 typical samples are gathered for use in training and testing the BP network, 16 of which are randomly chosen as training patterns and the others are chosen as testing patterns, shown in Table1. These data basically contain the various distinct characteristics of the problem the BP network is likely to encounter in the finished application. Preprocessing of the data is usually required before presenting the patterns to the BP network. This is necessary because the sigmoid activation function modulates the output of each neuron to values between 0 and 1 . The prediction results shown in Table2.

Table 1 The samples of the BP neural network mode 


\begin{tabular}{|c|c|c|c|c|c|}
\hline $\begin{array}{c}\text { Serial } \\
\text { number }\end{array}$ & $\begin{array}{c}\text { Cutting } \\
\text { pressure } \\
(\mathrm{MPa})\end{array}$ & $\begin{array}{c}\text { Feed } \\
\text { rate } \\
(\mathrm{mm} / \mathrm{min})\end{array}$ & $\begin{array}{r}\text { Additive } \\
\text { concentration }\end{array}$ & $\begin{array}{c}\text { Standoff } \\
\text { distance } \\
(\mathrm{mm})\end{array}$ & $\begin{array}{c}\text { Cutting } \\
\text { depth }(\mathrm{mm})\end{array}$ \\
\hline 1 & 15 & 15 & $1: 10$ & 2 & 12.1 \\
\hline 2 & 15 & 30 & $1: 10$ & 2 & 8.6 \\
\hline 3 & 15 & 60 & $1: 10$ & 2 & 4.5 \\
\hline 4 & 15 & 120 & $1: 10$ & 2 & 2.1 \\
\hline 5 & 20 & 15 & $1: 12$ & 4 & 14.8 \\
\hline 6 & 20 & 30 & $1: 12$ & 4 & 10.3 \\
\hline 7 & 20 & 60 & $1: 12$ & 4 & 6.6 \\
\hline 8 & 20 & 120 & $1: 12$ & 4 & 3.2 \\
\hline 9 & 25 & 15 & $1: 14$ & 6 & 20.1 \\
\hline 10 & 25 & 30 & $1: 14$ & 6 & 12.5 \\
\hline 11 & 25 & 60 & $1: 14$ & 6 & 7.4 \\
\hline 12 & 25 & 120 & $1: 14$ & 6 & 5.3 \\
\hline 13 & 30 & 15 & $1: 16$ & 8 & 23.2 \\
\hline 14 & 30 & 30 & $1: 16$ & 8 & 15.5 \\
\hline 15 & 30 & 60 & $1: 16$ & 8 & 9.4 \\
\hline 16 & 30 & 120 & $1: 16$ & 8 & 7.3 \\
\hline 17 & 15 & 15 & $1: 16$ & 2 & 13.1 \\
\hline 18 & 20 & 30 & $1: 14$ & 4 & 9.8 \\
\hline 19 & 25 & 60 & $1: 12$ & 6 & 7.1 \\
\hline 20 & 30 & 120 & $1: 10$ & 8 & 6.9 \\
\hline
\end{tabular}

Table 2 prediction results of the BP neural network model

\begin{tabular}{|c|c|c|c|c|}
\hline $\begin{array}{c}\text { serial } \\
\text { number }\end{array}$ & $\begin{array}{c}\text { Measured } \\
\text { depth }(\mathrm{mm})\end{array}$ & $\begin{array}{r}\text { Predicted } \\
\text { depth }(\mathrm{mm})\end{array}$ & $\begin{array}{r}\text { Absolute } \\
\text { discrepancy }(\mathrm{mm})\end{array}$ & $\begin{array}{c}\text { Relative } \\
\text { discrepancy } \\
(\%)\end{array}$ \\
\hline 17 & 13.1 & 13.7 & 0.6 & 4.58 \\
\hline 18 & 9.8 & 9.6 & -0.2 & 2.04 \\
\hline 19 & 7.1 & 7.4 & 0.3 & 4.23 \\
\hline 20 & 6.9 & 6.3 & -0.6 & 8.70 \\
\hline
\end{tabular}

\section{CONCLUSIONS}

A BP neural network can predict the cutting depth generated by abrasive suspension jet, and the prediction results show greater accuracy than those from the traditional statistical models. The modeling process based on the BP neural network is much easier and direct, for there is no necessity to specify a mathematical relationship between the input and output variables. The BP neural network can be effective for analyzing a system containing a number of variables, to establish patterns and characteristics not previously known. However, there are some problems need to be well discussed in the applications of BP neural network, such as the choice of the preset training precision and the proper number of hidden neurons etc. 


\section{ACKNOWLEDGEMENTS}

The authors wish to acknowledge the funding by the Science Technology Committee of Shanghai .Acknowledgement is also due to Professor Liu Linsheng who provided help in the test. The authors are indebted to Dr. Wang Wenbin, Hu guihua for their help in the investigation.

\section{REFERENCES}

1.Xiao-jian Liu Tao Yu ,Study on the Theory of A New Kind of Abrasive Jet Cutting Technology and the Test Analyses, Machinery, 2005.2.

2. Liu Xiaojian, Yu Tao, Liu Linsheng Wang Wenbin 'The study on abrasive suspension jet cutting technology", Lubrication Engineering, 2005,169(3), 31-33,

3. Cybenko, G., Mathematics of Control Signal and Systems, 2003, 2, 303.

4. J.M. Cabrera, A. Alomar, J.J. Jonas, J.M. Prado, Metall. Trans. A 28 (1997) 2233-2244.

5. Taylor. J.G.(1998) Neural Networks :an overview in L.J. Landau, \&J.F. Taylor. Concepts for neural networks - a Survey London :Springer.

7. G. A. Carpenter and S. Huang, The ART of adaptive pattern recognition by self-organizing neural networks, Computer $2004,22(6), 77-88$.

8. L. Monostori and D. Barschdorff, Artificial neural networks in intelligent manufacturing, Robotics and Computer Integrated Manufacturing 2002,9(2),412-436.

9. Refenes A. N. Stock performance modeling using neural networks : A Comparative study with regression models, Neural Networks, 1994,17 (2).36-48. 